\title{
Low Cost and Open Source Purification Apparatus for GMP $\left[{ }^{13} \mathrm{~N}^{\mathrm{NH}} \mathrm{H}_{3}\right.$ Production
}

Farah Kamar, ${ }^{1}$ Michael S. Kovacs, ${ }^{1,2,3}$ Justin W. Hicks ${ }^{1,3}$

1) Medical Biophysics, Western University, London, ON, Canada

2) Medical Imaging, Western University, London, ON, Canada

3) Cyclotron and Radiochemistry Facility, Lawson Health Research Institute, London, ON, Canada

\begin{abstract}
Nitrogen-13 labeled ammonia $\left(\left[{ }^{13} \mathrm{~N}^{-} \mathrm{NH}_{3}\right)\right.$ has been used for myocardial perfusion imaging with Positron Emission Tomography for decades. Recent increases to regulatory oversight have led to stricter adherence Good Manufacturing Practice (GMP) to produce this short half-life (9.97 min) radiopharmaceutical. This has increased production costs. Our cyclotron facility initially developed a manual GMP production method, but it was prone to human error. With increased costs in mind, we developed and validation an Arduino-based device to purifying $\left[{ }^{13} \mathrm{~N}^{\mathrm{N} H} \mathrm{H}_{3}\right.$ for clinical use. Construction, programming, and GMP validation results are discussed. The automated method was found to produce equivalent quality radiopharmaceutical but was more reproducible and robust.
\end{abstract}

\section{Introduction}

Radiopharmaceutical production is challenging even for the simplest molecules. Common radionuclides used for Positron Emission Tomography (PET) have short half-lives and emit high levels of radiation. These factors necessitate rapid production methods in a shielded, controlled environment. Additionally, if these imaging agents are destined for human administration, they must be produced in an aseptic environment and tested for release as any other pharmaceutical. These constraints have encouraged automating routine radiopharmaceutical production. For our purposes, we sought a robust automation of nitrogen-13 $\left(t_{1 / 2}=9.97 \mathrm{~min}, \beta^{+}=100 \%, E_{\max } B^{+}=1.19 \mathrm{MeV}\right)$ labeled ammonia production for use in PET myocardial perfusion imaging. ${ }^{1-3}$ 
Approximately 1 in 12 Canadian adults 20 years of age and older live with diagnosed coronary heart disease with coronary artery disease (CAD) making up most cases. ${ }^{4}$ CAD leads to stiffer and narrower coronary arteries which can result in hypoxia and ischemia of the cardiac muscles. This deficiency of oxygen and nutrients in cardiac muscles makes it difficult for the heart to pump and transport blood around the body. Decreased perfusion of the cardiac muscles can lead to angina or a myocardial infarction (MI). It is, therefore, crucial to monitor those with CAD to allow early diagnosis and guide treatment to prevent further progression. ${ }^{5}$ While $\left[{ }^{13} \mathrm{~N}\right] \mathrm{NH}_{3}$ is an excellent perfusion biomarker, it's short half-life requires a well coordinated production, often timed to coincide with stress testing. ${ }^{3}$ Reliable, on-demand production methods are therefore required to meet clinical needs.

Production methods involved 1) reducing the $\left[{ }^{13} \mathrm{~N}\right] \mathrm{NO}_{x}$ species from the cyclotron target to $\left[{ }^{13} \mathrm{~N}^{-N_{\mathrm{NH}}} \mathrm{H}_{3}\right.$ using $\mathrm{TiCl}_{3}$ or DeVarda's alloy, or 2) direct production within the target using additives such as ethanol, hydrogen, or methane to prevent oxidation. These methods have been recently reviewed elsewhere. ${ }^{8}$ For simplicity post-bombardment, in-target production $^{9}$ is preferred. . One simple and effective method passes the target solution through a quaternary ammonium cartridge with $\left[{ }^{13} \mathrm{~N}^{-N_{\mathrm{NH}}}\right.$ trapping onto a cationic capture cartridge in series. ${ }^{10}$ This removes any ${ }^{13} \mathrm{~N}$-labeled anionic species as well as any potential $\left[{ }^{18} \mathrm{~F}\right]$ fluoride from small amounts of ${ }^{18} \mathrm{O}$ in the target solution. The product is then selectively eluted with sterile saline through a $0.22 \mu \mathrm{m}$ sterile filter. These steps require actuating a valve by hand or through automation. If radiation exposure was not a concern, then simply turning two stopcocks would suffice. Manipulator arms could be used to rotate disposable stopcocks if an operator with adequate skill is available. This was how we initially produced $\left[{ }^{13} \mathrm{~N}^{\mathrm{N} H} \mathrm{~N}_{3}\right.$ for 3 years of clinical studies.

As one can imagine, turning the stopcocks with manipulator arms was not an easy task. There were many instances where the tubing would snag and disconnect or turning the stopcock would accidently pull the apparatus apart. Ergonomically, it was a strain on the operator to conduct such fine motor 
movements repeatedly, leading to increased errors when multiple batches were produced in a single day. To improve reliability, we sought a means to automate the $\left[{ }^{13} \mathrm{~N}\right] \mathrm{NH}_{3}$ production.

From the advent of PET radiochemistry, in-house automated apparatuses have been assembled to handle the transfer, heating, and purification during productions. ${ }^{11,12}$ To construct and maintain these, a high degree of electrical engineering and programing experience were required. Various commercial instruments are now available, but these are expensive and often cumbersome to customized productions. For the simplest productions of very short half-life radiopharmaceuticals such as $\left[{ }^{13} \mathrm{~N}^{\mathrm{N} H} \mathrm{H}_{3}\right.$, these commercial systems are overly complicated and costly.

Recent availability of easy to assemble and program microprocessors, such as Arduino or Raspberry $\mathrm{Pi}$, have improved access to the non-initiate. As radio-chemists or -pharmacists, these inexpensive devices can be assembled through many online tutorials from a large community of do-it-yourself scientists and creators, without needing a background in electrical engineering. Open-source programming also permits a novice with basic programming knowledge to program code to operate these homemade devices. One recent example developed an elegant device for producing $\left[{ }^{11} \mathrm{C}\right]$ acetate without having to alter existing infrastructure. ${ }^{13}$

As one of the oldest clinically used radiopharmaceuticals, $\left[{ }^{13} \mathrm{~N}^{\mathrm{N} H} \mathrm{H}_{3}\right.$ costs have historically been low. As more regulatory burden has been placed on its production, there has been upward pressure on price to recover production costs. To maintain the low cost of this simple positron-emitting radiopharmaceutical, we sought to avoid commercially available devices in favour of constructing an Arduino-based valve controller. The construction and validation of this apparatus will be discussed below. 


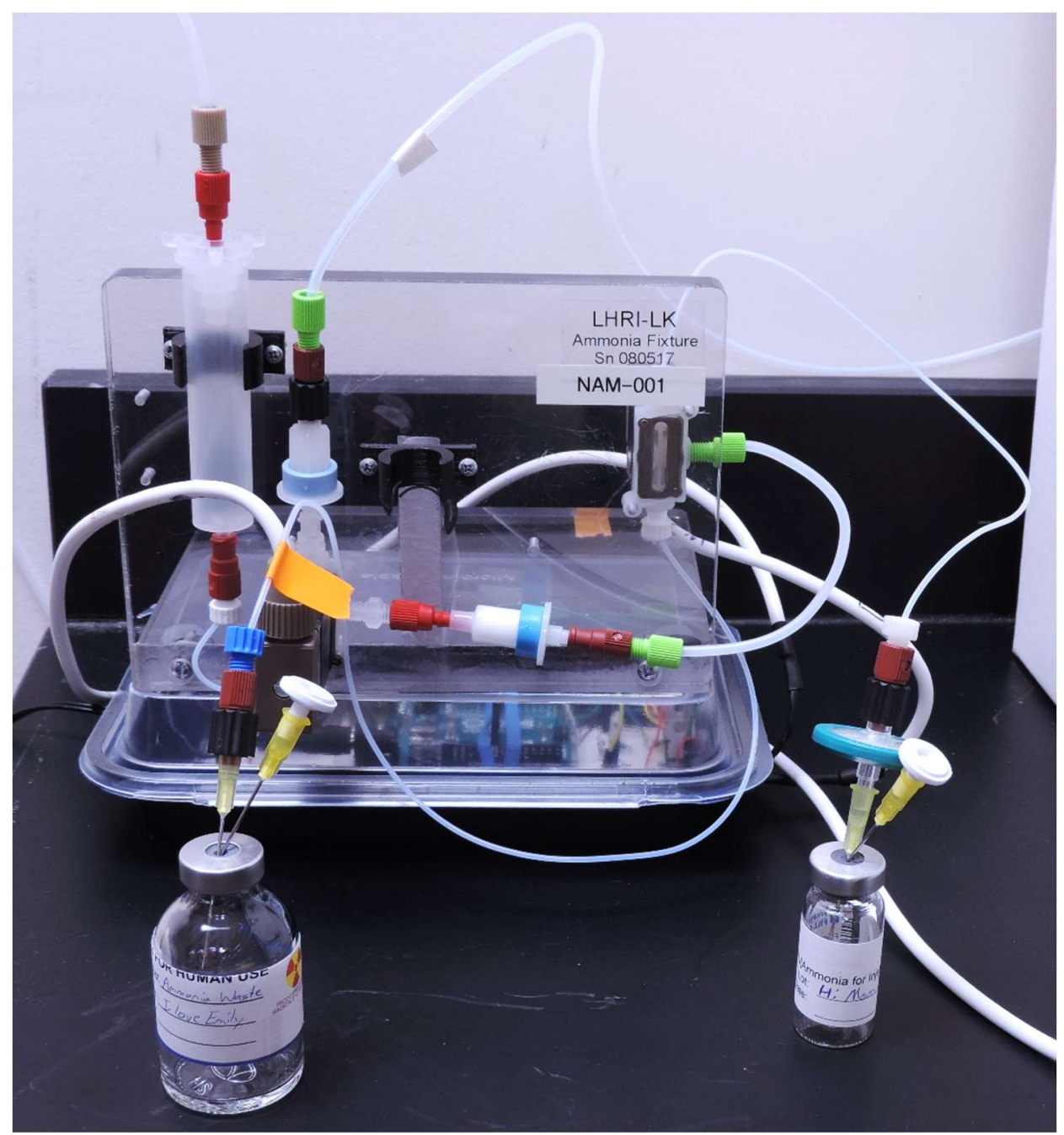

Figure 1. The Nitrogen-13 Ammonia Manufacturing (NAM) device assembled to automatically purify $\left[{ }^{13} \mathrm{~N}^{-N_{3}} \mathrm{H}_{3}\right.$. The fluid pathway is on top of the electronics in encased in plastic. A $30 \mathrm{~mL}$ vial on left is for waste and the $10 \mathrm{~mL}$ vial on right is the final product vial.

\section{Method and Materials:}

\section{Production and Purification of $\left[{ }^{13} \mathrm{~N}\right] \mathrm{NH}_{3}$}

A $10 \mathrm{mM}$ ethanol solution in ultra low conductance water (4 mL; Millipore Omnitrace Ultra water; WX0003) was bombarded with a $40 \mu \mathrm{A}$ proton beam for 10 min using an $18 \mathrm{MeV}$ GE PETtrace. The nitrogen-13 target effluent flowed from the cyclotron through two ion exchange cartridges. A Waters QMA chloride (WAT023525) cartridge captured the anionic impurities and a Waters Accel CM (WAT020550) cartridge trapped the $\left[{ }^{13} \mathrm{~N} \mathrm{NH}_{3}\right.$. The remaining fluid flowed into a waste container. Upon complete delivery of the target contents, the valves (manual stopcocks or solenoids) were 
switched to secondary positions. In this second valve position (Figure 3b), the QMA cartridge was bypassed and nitrogen driven saline flowed through the Accel $\mathrm{CM}$ cartridge to elute the $\left[{ }^{13} \mathrm{~N}\right] \mathrm{NH}_{3}$ through a sterile filter (Millipore Millex-GP PES $33 \mathrm{~mm}, 0.22 \mu \mathrm{m}$; SLGP033RS) into a final product vial.

\section{Electronic Assembly}

The three main components were the Arduino UNO, a protoboard, and solenoid valves (Figure 2). A triggering button (Judco Manufacturing 50-00015-00) was wired into the UNO (Arduino A000073) position 2 and ground on the protoboard (Twin Industries TW-E41-1020). The gate terminal of two Nchannel MOSFET (ON semiconductors FDP8447-L) transistors were connected to position 3 and 5 while the drain was connected to the solenoid (Burkert $01273 / 2$ rocker valve), and the source to ground. Note the connected diode (ON semiconductor 1N5934BG) circuit to the MOSFET drain. This diode prevents damage to the circuit from built up charge in the solenoid. Positions 11 and 12 of the UNO were connected to the LED lights through 221ohm resistors (Yageo MFR-25FBF52-221R). Two red light emitting diodes (LED; Cree Inc. C503B-RAN-CZ0C0AA1) were installed as visual cues for triggering the solenoid valves. One light was installed per solenoid as two sets which were wired in parallel. A 24V plug-in AC/DC power supply (Triad Magnetics WSU240-0500) was connected to the protoboard. The solenoid valves required $24 \mathrm{~V}$ to actuate but this voltage is too high for the UNO microcontroller. To avoid using two power sources, a 12V DC-DC converter (Traco Power TMV $2412 \mathrm{SHI}$ ) was used to lower the voltage to power the UNO. This converter was connected to the Arduino Uno through the power port. Jumper wires used were from Adafruit Industries (part number 153). A close-up image of the constructed circuit is found in Figure 5. 


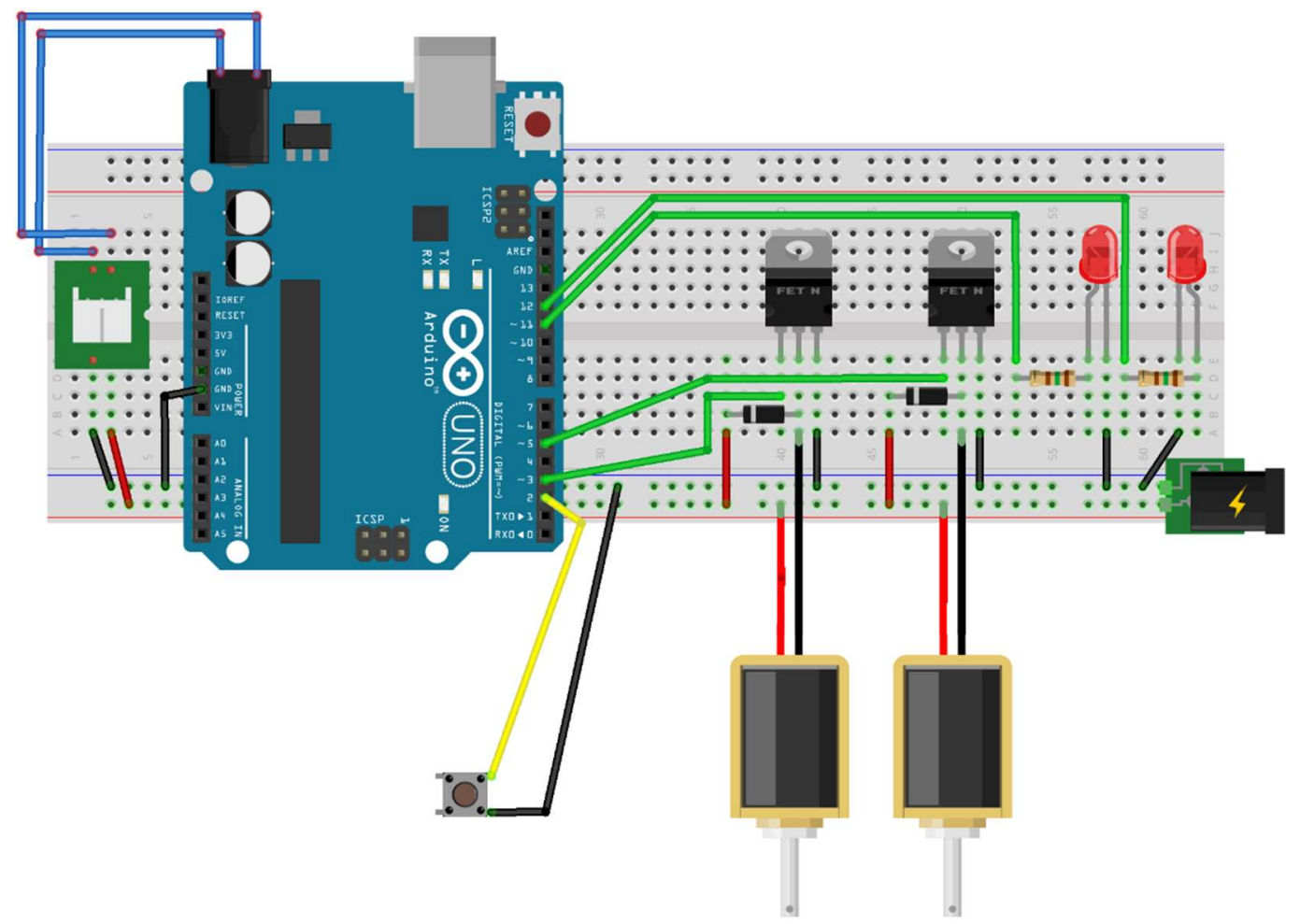

Figure 2. Electronic schematic drawn using Fritzing (version 0.9.9). ${ }^{14}$

\section{Programming}

The code used to control the microcontroller is in Figure 3 . The setup section sets the initial state of the pins while the loop is the main logic. In the setup, the button is set as an input, and the valves and LEDs are set as outputs. The loop code detects the button status by checking if the circuit path is closed to the Arduino Uno from the power supply. This code repeats on a cycle to constantly check the button state to switch the valves off if the incoming current path is opened by hitting the button once more which switched the solenoid valves back to position 1 (Figures 4 and 5). 


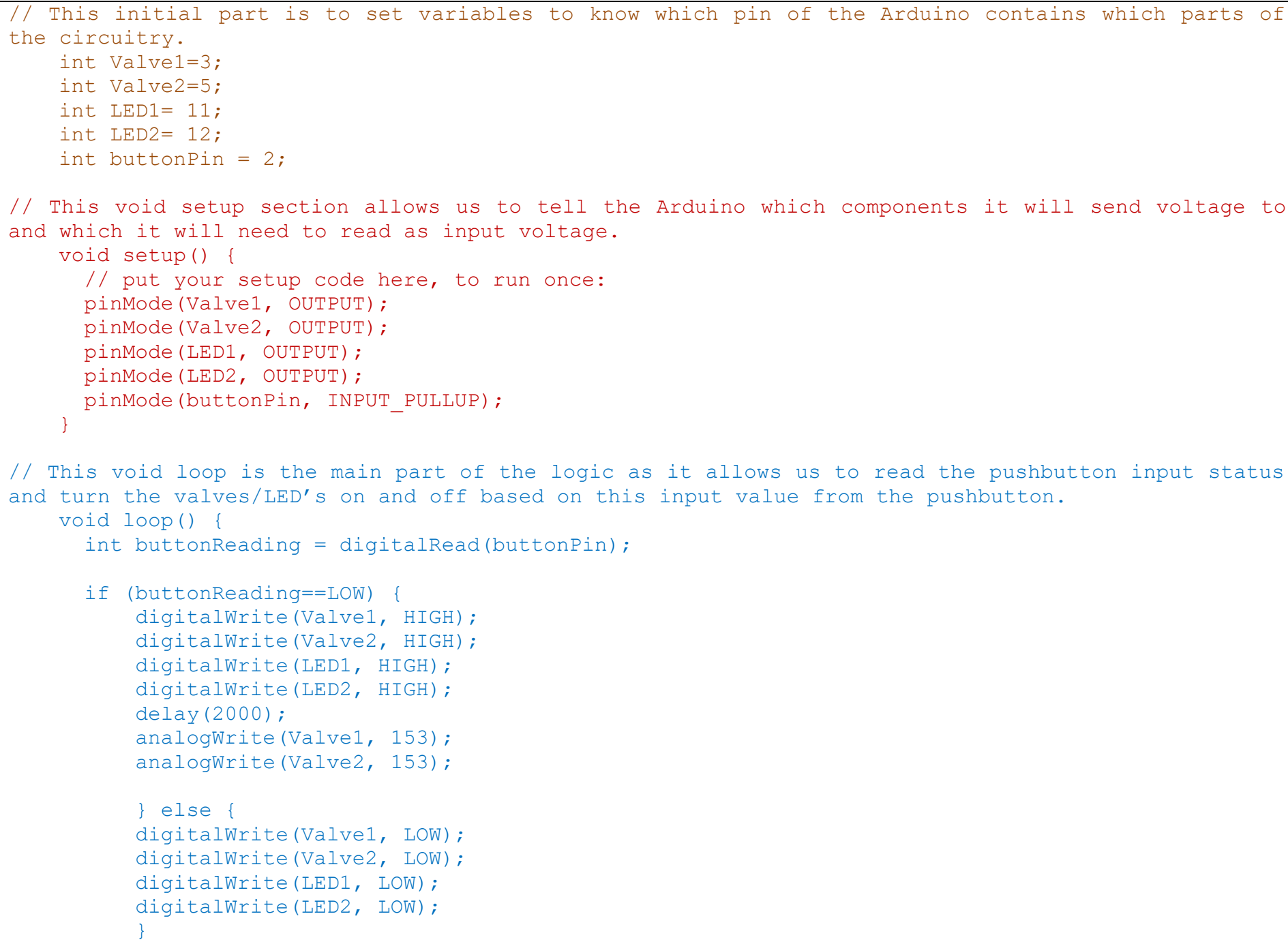

Figure 3 - Arduino code for operating the automated, two valve apparatus for purifying $\left[{ }^{13} \mathrm{~N}^{\mathrm{N}} \mathrm{NH}_{3}\right.$

\section{Fluid pathway}

The cyclotron target outlet was connected to the top of the QMA chloride cartridge which was placed onto the common position of the first solenoid valve (Figure 4). The normally open (NO) outlet was connected to the common position of the second solenoid while the normally closed position is attached to a reservoir capable of holding $8 \mathrm{~mL}$ of sterile saline. The second solenoid valve is connected to a waste container (NO) and the final product vial (NC). The apparatus was cleaned with $8 \mathrm{~mL}$ of water and ethanol then dried with nitrogen between each production. A machined holder was manufactured in-house. 


\section{Validation protocol}

Two preliminary doses of $\left[{ }^{13} \mathrm{~N} \mathrm{NH}_{3}\right.$ are prepared for sterility and quality control testing, respectively, prior to preparing patient doses. This limits septum punctures and allows for sterility testing of the first batch produced, which is at greatest risk as repeated high activity deliveries will flush and sterilize the fluid pathway. For production validation, additional quality control testing was conducted on the final dose produced to bracket testing. All doses in a production batch were prepared in an identical manner and assumed to meet the same release specification as those undergoing quality testing. All testing, as outlined in product monographs, ${ }^{10}$ were validated in house with results tabulated in Table 1.

\section{Results:}

Originally to keep costs to a minimal, a manual apparatus was developed by our prototyping facility to hold the cartridges and stopcocks. This was adequate to validate the GMP production of $\left[{ }^{13} \mathrm{~N}^{\mathrm{N} \mathrm{NH}_{3}}\right.$ (results in Table 1) but was prone to operator errors. These necessitated the repetition of quality control and/or patient dose productions, or the complete failure to release $\left[{ }^{13} \mathrm{~N}^{-N} \mathrm{H}_{3}\right.$ for patient injection. Both setbacks resulted in wasted resources without cost recovery and the delay of patients' important PET imaging results. Thus, we endeavoured to construct an automated device.

The circuitry (Figures 2 and 5) and plumbing (Figures 1 and 4) were successfully assembled in the hot cell with the button accessible from outside or placed inside (can press with manipulator arms) After uploading and testing the operating code to the Arduino board, the performance of the apparatus was tested. As intended, the valves switched when the pushbutton was pressed. The LED lights were on when the valves were in position 2 and off at valve position 1 following a second button push (Figure 5). For a typical 10 min, $40 \mu \mathrm{A}$ bombardment we will obtain roughly $10 \mathrm{GBq}$ of $\left[{ }^{13} \mathrm{~N}^{-N_{\mathrm{NH}}}\right.$ 
as the ${ }^{16} \mathrm{O}(\mathrm{p}, \mathrm{\alpha})^{13} \mathrm{~N}$ reaction produces $1788 \pm 181 \mathrm{MBq} / \mu \mathrm{Ah}$. In a preliminary test with the automated device, we obtained 9.4 GBq at end of synthesis (EOS).

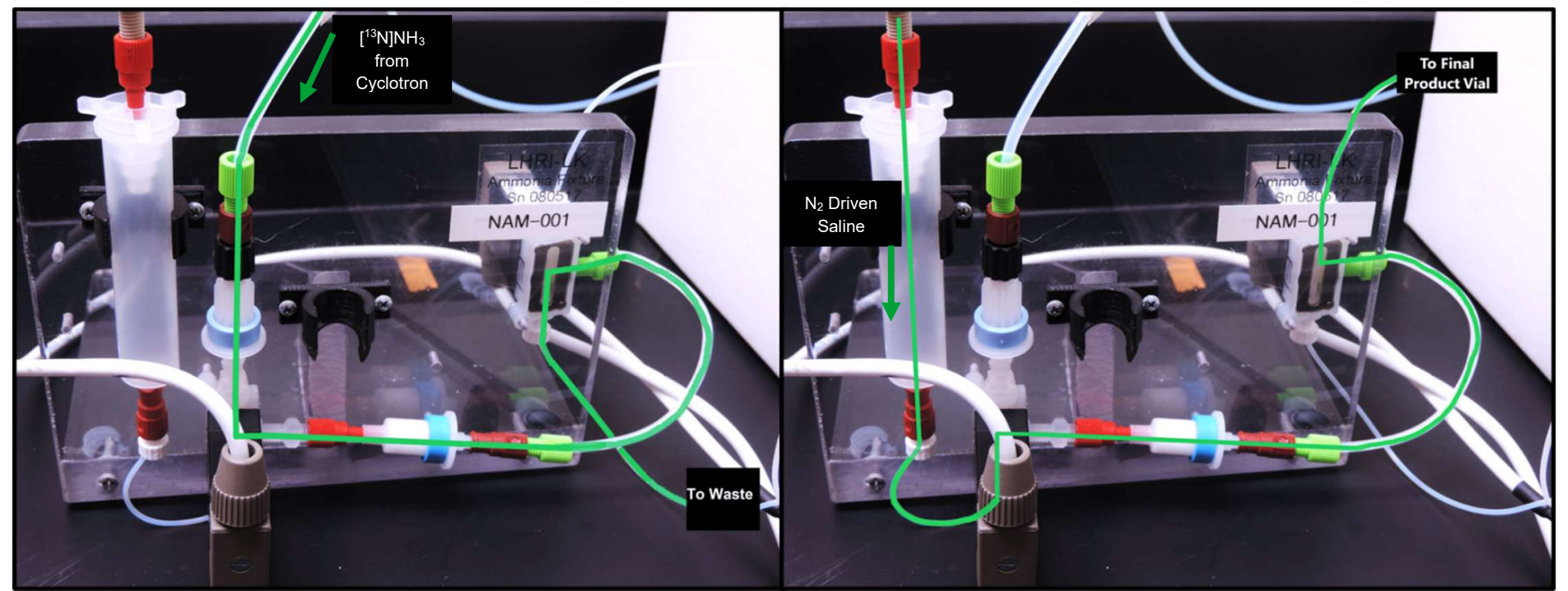

Figure 4. Fluid pathway connections with the solenoid valves in initial (left) and triggered (right) positions. Overlaid green lines illustrate fluid path.

We have previously validated the manual purification method and produced dozens of $\left[{ }^{13} \mathrm{~N}^{-N_{3}} \mathrm{H}_{3}\right.$ doses over the two-year period that we were supplying the clinical PET instrument. Due to the short half-life of ${ }^{13} \mathrm{~N}$ and our physical setup, it was more practical to produce preliminary batches for sterility and quality control (QC) testing than to rush testing for release of human doses. Our protocol was to first obtain a sterility sample as this represented the greatest risk due to delivery lines sitting idle. The high concentration of ionizing radiation and pressure from target delivery would likely clear the lines initially if anything was present. As we have not observed any positive growth in our sterility testing, this hypothesis remains valid although no positive control experiments have been attempted.

A QC batch is produced second prior to preparing any human doses. This allows for QC staff to comfortably complete testing without rushing. A second QC sample was tested from the final batch produced, thus bracketing all doses with quality control. Initially this was the sixth patient dose (eighth batch overall), but we found demand was not that high. We switched to a two-patient dose 
qualification for the automated purification (four batches in total). If on a rare occasion more than two $\left[{ }^{13} \mathrm{~N} \mathrm{NH}_{3}\right.$ doses were required, we would have to produce another sterility and QC batch for the third and fourth doses.
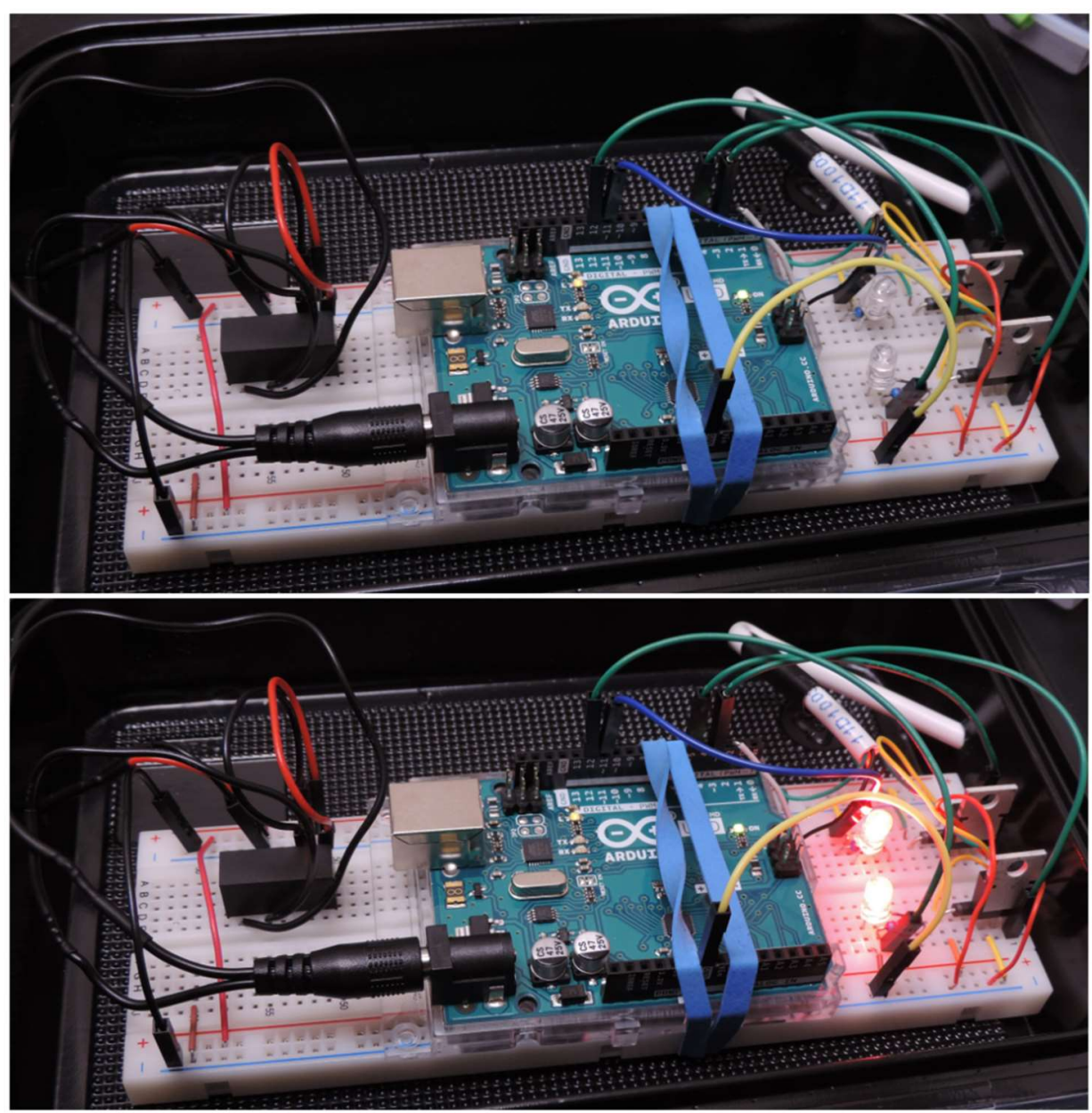

Figure 5. Close up of circuit before (left) and after (right) triggering the device via a push button.

Testing results are summarized in Table 1. For manual and automated methods, we obtained $11.9 \pm$ 1.3 and $13.7 \pm 3.6 \mathrm{GBq}$ at EOS, respectively. We found no difference between the two methods regarding $\left[{ }^{13} \mathrm{~N}^{\mathrm{N}} \mathrm{NH}_{3}\right.$ quality. Importantly from a patient safety standpoint, there were no failing results with sterility, endotoxin levels, or residual ethanol related to the reusable valves and tubing. The only major difference was a lack of production failures due to human error. On average, the manual purification method would fail roughly once every seven batches produced. When considering that a minimum of three batches are required, that translated into almost every third patient missing their 
dose. Common causes of failure were disconnected tubing (caught on manipulator arms or twisted off with turning stopcock) or errors assembling the disposable stopcocks and tubing. Improvements to reproducibility was a main objective to this work. Anecdotally, the production staff has mentioned improved ergonomic comfort without having to turn stopcocks with the manipulator arms. Safe and happy production staff is an additional benefit of automating the valve switching with a button push.

Table 1. Results from GMP production validation protocols

\begin{tabular}{cccc}
\hline Specification & Release Criteria & Manual & Automated \\
\hline \hline Radioactivity & $N L T 740 \mathrm{MBq} / \mathrm{mL}$ at EOS & $1485 \pm 159$ & $1831 \pm 69$ \\
Concentration & $9.47<t_{1 / 2}<10.47$ min & $9.73 \pm 0.04$ & $9.98 \pm 0.24$ \\
Radionuclidic Identity & NLT 95\% & $1.6 \pm 0.6 \%$ & $1.8 \pm 0.1 \%$ \\
Radiochemical Identity & $t_{R} N M T 10 \%$ from standard & $99.6 \pm 0.2 \%$ & $99.9 \pm 0.1 \%$ \\
Radiochemical purity & $N L T 99.5 \%$ gamma emissions & $100 \%$ & $100 \%$ \\
Radionuclidic purity & corresponding to 511 keV & $6.6 \pm 1.2$ & $7.4 \pm 0.2$ \\
pH & $4.5-7.5$ & $3 \pm 2$ & $4 \pm 3$ \\
Residual Ethanol & $N M T 5000$ ppm & $<2.5$ & $<2.5$ \\
Bacterial Endotoxin & $N M T 21.9$ EU/mL & Conforms & Conforms \\
Appearance & Clear, colourless, particulate free & Conforms & Conforms \\
Sterility & No growth & 104 & 12 \\
\hline
\end{tabular}

$\mathrm{NLT}=$ no less than, NMT $=$ no more than, $\mathrm{EOS}=$ end of synthesis, $\mathrm{t}_{\mathrm{R}}=$ retention time, $\mathrm{EU}=$ endotoxin units

Due to the COVID-19 global pandemic, $\left[{ }^{13} \mathrm{~N} \mathrm{NH}_{3}\right.$ scans have not yet been produced for patient using this automated procedure. This has limited our data to solely the production validation $(n=12)$. Additionally, the project protoboard used to create the circuit is crude and should be improved with a proper Arduino shield. Given the exposure to high levels of ionizing radiation, a lead shielded case will be constructed such that the Arduino device can remain in the hot cell. Currently, it is removed between productions. Coupled with the protoboard construction, this removal introduces opportunities for disconnections within the circuit. Our code could also be refined to include pulse width modulation once the solenoid valves are activated. This would only supply enough current to keep the solenoids 
energized as full current generates significant heat, leading to increased wear on the solenoid and accelerating component failure. Lastly, while the reliability of a physical button is unparalleled, a wireless trigger (potentially a smart phone app) is sought to further minimize the wired connections.

\section{Conclusion}

This low-cost, easily assembled device was able to reliably produce $\left[{ }^{13} \mathrm{~N}\right] \mathrm{NH}_{3}$ to cGMP standards. When compared to our standard manual procedure, the automated Arduino-based device prerformed as well in terms of product quality with fewer failures. For less than $\$ 100$, a compact device was assembled and programmed. With limited experience, the barrier to building this was quite low. The large, open-source community of DIY-ers permits immense possibilities to improve and expand upon this initial build. This simple production does not require sensors or a user interface, but these could be easily incorporated. More use of these open-source tools will lower access barrier to automating radiochemistry procedures, freeing tight budgets for conducting more innovative research.

\section{Author Contributions}

FK conceived, constructed, and beta tested the Arduino device. MSK oversaw cyclotron facility and staff. JWH conceived of the project, produced the $\left[{ }^{13} \mathrm{~N}^{\mathrm{N} \mathrm{NH}_{3}}\right.$, and oversaw all validation testing. All authors provided input in the writing of the manuscript.

\section{Acknowledgements}

The authors would like to thank Lynn Keenliside in the Lawson Prototyping Facility for early guidance and discussions. For the assistance with the quality control testing, we are eternally grateful to Amanda Rodrigues, Sandra Penner, and Chloé Palesh. We also thank Neil Cockburn for helpful discussions surround cGMP validation. We are grateful for funding provided by Western's Schulich School of Medicine and Dentistry as part of FK's fourth year undergraduate project. GE Healthcare supplied the ${ }^{13} \mathrm{~N}$ target system as an in-kind contribution. 


\section{References}

(1) Schindler, T. H.; Schelbert, H. R.; Quercioli, A.; Dilsizian, V. Cardiac PET Imaging for the Detection and Monitoring of Coronary Artery Disease and Microvascular Health. JACC Cardiovasc. Imaging 2010, 3 (6), 623-640. https://doi.org/10.1016/j.jcmg.2010.04.007.

(2) Cardiac System. In Nuclear Medicine (Fourth Edition); Ziessman, H. A., O'Malley, J. P., Thrall, J. H., Eds.; W.B. Saunders: Philadelphia, 2014; pp 378-423. https://doi.org/10.1016/B978-0323-08299-0.00016-X.

(3) Schindler, T. H.; Bateman, T. M.; Berman, D. S.; Chareonthaitawee, P.; Blanche, L. E. D.; Dilsizian, V.; Dorbala, S.; Gropler, R. J.; Shaw, L.; Soman, P.; Winchester, D. E.; Verberne, H.; Ahuja, S.; Beanlands, R. S.; Carli, M. F. D.; Murthy, V. L.; Ruddy, T. D.; Schwartz, R. G. Appropriate Use Criteria for PET Myocardial Perfusion Imaging. J. Nucl. Med. 2020, 61 (8), 1221-1265. https://doi.org/10.2967/jnumed.120.246280.

(4) Public Health Agency of Canada (PHAC). Heart Disease in Canada https://www.canada.ca/en/public-health/services/publications/diseases-conditions/heartdisease-canada.html (accessed $2021-10$-20).

(5) Jia, S.; Liu, Y.; Yuan, J. Evidence in Guidelines for Treatment of Coronary Artery Disease. Adv. Exp. Med. Biol. 2020, 1177, 37-73. https://doi.org/10.1007/978-981-15-2517-9_2.

(6) Krizek, H.; Lembares, N.; Dinwoodie, R.; Gloria, I.; Lanthrop, K.; Harper, P. Production of Radiochemically Pure $13 \mathrm{NH} 3$ for Biomedical Studies Using the $16 \mathrm{O}(\mathrm{p}, \alpha) 13 \mathrm{~N}$ Reaction. J. Nucl. Med. 1974, 14, 629-630.

(7) Vaalburg, W.; Kamphuis, J. A. A.; Beerling-van der Molen, H. D.; Reiffers, S.; Rijskamp, A.; Woldring, M. G. An Improved Method for the Cyclotron Production of 13N-Labelled Ammonia. Int. J. Appl. Radiat. Isot. 1975, 26 (5), 316-318. https://doi.org/10.1016/0020-708X(75)90169-6.

(8) Tsai, C.-L.; Huang, Y.-Y.; Tang, K.-H.; Wen, H.-P.; Tzen, K.-Y.; Shiue, C.-Y.; Yen, R.-F. Comparison of [N-13]NH3 Production Methods: Practical Experience of National Taiwan University Hospital. Ann. Nucl. Med. Mol. Imaging 2018, 31, 199-207. https://doi.org/10.6332/ANMMI.201812_31(4).0001.

(9) Berridge, M. S.; Landmeier, B. J. In-Target Production of [13N]Ammonia: Target Design, Products, and Operating Parameters. Appl. Radiat. Isot. 1993, 44 (12), 1433-1441. https://doi.org/10.1016/0969-8043(93)90096-S.

(10) Rice, P. A.; Yokell, D. L. Determination of Radiochemical Purity and Radiochemical Identity of [13N]NH3 Using Thin Layer Chromatography. In Radiochemical Syntheses; John Wiley \& Sons, Ltd, 2015; pp 241-246. https://doi.org/10.1002/9781118834114.ch23.

(11) Decristoforo, C. Challenges in the Small-Scale Preparation of Radiopharmaceuticals - A European Perspective. FABAD J. Pharm. Sci. 2007, 32, 131-138.

(12) Alexoff, D. L. Automation for the Synthesis and Application of PET Radiopharmaceuticals. In Handbook of Radiopharmaceuticals; John Wiley \& Sons, Ltd, 2002; pp 283-305. https://doi.org/10.1002/0470846380.ch8.

(13) Maurer, A.; Bowden, G.; Cotton, J.; Parl, C.; Krueger, M. A.; Pichler, B. J. Acetuino-A Handy Open-Source Radiochemistry Module for the Preparation of [1-11C]Acetate. SLAS Technol. Transl. Life Sci. Innov. 2019, 24 (3), 321-329. https://doi.org/10.1177/2472630318812341.

(14) Fritzing http://fritzing.org/ (accessed $2021-10$-21). 